\title{
A SPLITTING RING OF GLOBAL DIMENSION TWO ${ }^{1}$
}

\author{
JOHN D. FUELBERTH AND MARK L. TEPLY
}

\begin{abstract}
In this paper an example is given of a ring with left global dimension 2 having the property that the singular submodule of any $R$-module $A$ is a direct summand of $A$. Although the example given is quite specific, the methods can be used to construct a fairly large class of these rings.
\end{abstract}

In this paper, all rings are assumed to be associative with an identity element, and all modules will be unitary left modules.

An $R$-module $A$ is said to split if the singular submodule, $Z\left({ }_{R} A\right)$, is a direct summand of $A . R$ is called a splitting ring if every $R$-module splits (see [1], [3], and [7]). In [3] Cateforis and Sandomierski have shown that every commutative splitting ring has left global dimension $\leqq 1$. M. L. Teply [7] has shown that if the commutative hypothesis is dropped, then every splitting ring must have left global dimension $\leqq 2$. Several splitting rings of left global dimension 1 were known, but no such rings of left global dimension 2 have been found; thus the question arises, which is the best bound, 1 or 2 ? In this paper it is shown that 2 is the best possible bound for the left global dimension of a splitting ring.

$R$ is said to have the finitely generated splitting property (FGSP) if every finitely generated $R$-module splits. Cateforis and Sandomierski [3] have shown that every commutative ring with FGSP must be semihereditary. A trivial consequence of the example constructed in this paper is that a (noncommutative) ring with FGSP need not be left or right semihereditary.

For an $R$-module $A$, let $\operatorname{soc}(A)$ denote the socle of $A$. If $R$ is a ring of matrices, we define $e_{i j}$ to be the matrix with the identity element of the appropriate coordinate ring in the $i$ th row and $j$ th column and zeros elsewhere.

Received by the editors July 26, 1971.

AMS 1970 subject classifications. Primary 18E40, 16A48; Secondary 16A14, 16A60, 16A62, $16 \mathrm{~A} 64$.

Key words and phrases. Singular module, nonsingular module, left global dimension, splitting ring, idealizer, simple module, injective module, hereditary Noetherian domain.

1 This work was partially supported by a University of Florida Graduate School Postdoctoral Fellowship. 
Let $S$ be a ring, and let $M$ be an essential maximal left ideal of $S$ which is also a two-sided ideal. Let

and let

$$
R=\left\{\left(\begin{array}{lll}
a & b & c \\
0 & d & e \\
0 & 0 & f
\end{array}\right) \mid d \in S ; a, b, c, e, f \in S / M\right\}
$$

$$
\Lambda=\left\{\left(\begin{array}{ccc}
a & 0 & 0 \\
0 & b & 0 \\
0 & 0 & c
\end{array}\right) \mid b \in S ; a, c \in S / M\right\}
$$

We wish to choose $S$ such that $R$ will become the desired splitting ring of left global dimension two. But first we point out a few basic properties of $R$ :

LEMma 1. (a) $R$ is a left (right) Noetherian ring if and only if $S$ is a left (right) Noetherian ring.

(b) If $Z\left({ }_{S} S\right)=0$, then $Z\left({ }_{R} R\right)=0$.

(c) If $S$ has no nontrivial idempotent elements, then $1 . g 1 . \operatorname{dim} R \geqq 2$.

Proof. (a) The "if" part is an immediate consequence of the fact that ${ }_{S} R$ is finitely generated. The "only if" follows from the existence of a (ring) homomorphism of $R$ onto $S$ given by

$$
\left(\begin{array}{lll}
a & b & c \\
0 & d & e \\
0 & 0 & f
\end{array}\right) \rightarrow d
$$

(b) If $Z\left({ }_{S} S\right)=0$, it is straightforward to check that annihilators of elements in the essential left ideal $R e_{11} \oplus R e_{22} \oplus R e_{13}$ are not essential in $R$. Hence $Z\left({ }_{R} R\right)=0$.

(c) If $1 . g l . d i m ~ R \leqq 1$, then

$$
\left(0: e_{23}\right)=\left\{\left(\begin{array}{lll}
a & 0 & b \\
0 & c & d \\
0 & 0 & e
\end{array}\right) \mid c \in M ; a, b, d, e \in S / M\right\}
$$

is generated by an idempotent element

$$
\left(\begin{array}{lll}
u & 0 & v \\
0 & w & x \\
0 & 0 & y
\end{array}\right)
$$

This forces $w^{2}=w \in M$, which contradicts the hypothesis that $S$ contains no nontrivial idempotent elements. 
Now let $C$ be a left and right principal ideal domain with the following properties:

(a) $C$ is a simple ring, which is not a division ring;

(b) every simple $C$-module is injective;

(c) there exists (up to isomorphism) only one simple $C$-module.

Examples of such rings have been provided by Cozzens [4]. Let $M$ be a maximal left ideal of $C$, and let $I=I_{C}(M)=\{x \in C \mid m x \in M$ for all $m \in M\}$ be the idealizer of $M$ in $C$ (see [6]). By [6, Theorem 4.3], $I$ is a hereditary Noetherian integral domain with nontrivial, two-sided ideal $M=M^{2}$. By [6, Theorem 1.3 and Corollary 2.4], $I$ has only two simple modules $S_{1}$ and $S_{2}$ (up to isomorphism); $S_{1}$ is a faithful injective simple module, $S_{2} \cong I / M$, and $E\left(S_{2}\right) / S_{2} \cong S_{1}$ (where $E\left(S_{2}\right)$ denotes the injective envelope of $S_{2}$ ). By [8, Theorem 4], every nonzero singular $I$-module has a nonzero socle.

We now give a sequence of lemmas designed to show that $I=S$ makes $R$ the desired splitting ring.

LEMMA 2. I is a splitting ring.

Proof. It is sufficient to show that $\operatorname{Ext}_{I}^{1}(F, T)=0$ for any nonsingular $I$-module $F$ and any singular $I$-module $T$.

As noted above, any singular $I$-module has nonzero socle; so $\operatorname{soc}(E(T))=\operatorname{soc} T$ is essential in $E(T)$. Write $\operatorname{soc}(T)=X \oplus Y$, where every simple submodule of $X$ is isomorphic to $S_{1}$ and every simple submodule of $Y$ is isomorphic to $S_{2}$. Since $S_{1}$ is injective and $I$ is Noetherian, then $X$ is injective and $E(T) \cong X \oplus E(Y)$. Moreover, either $E(Y)=0$ or else $E(Y)=$ $E\left(\oplus \sum_{\beta \in \mathscr{B}} S_{2}^{(\beta)} \cong \oplus \sum_{\beta \in \mathscr{B}} E\left(S_{2}^{(\beta)}\right)\right.$ for some index set $\mathscr{B}$, where $S_{2}^{(\beta)} \cong S_{2}$ for all $\beta \in \mathscr{B}$. Hence $\mathscr{B} \neq \varnothing$ implies

$$
E(T) / \operatorname{soc}(T) \cong \oplus \sum_{\beta \in \mathscr{B}}\left(E\left(S_{2}\right) / S_{2}\right) \cong \oplus \sum_{\beta \in \mathscr{B}} S_{1}^{(\beta)}
$$

with $S_{1}^{(\beta)} \cong S_{1}$ for all $\beta \in \mathscr{B}$. Since $I$ is hereditary and Noetherian, it follows that $E(T) / \operatorname{soc}(T)$ is injective. But $T / \operatorname{soc}(T)$ can be embedded (as a summand) in $E(T) / \operatorname{soc}(T)$; whence $T / \operatorname{soc}(T)$ is also injective. Hence we have the exact sequence

$$
\operatorname{Ext}_{I}^{1}(F, \operatorname{soc}(T)) \rightarrow \operatorname{Ext}_{I}^{1}(F, T) \rightarrow \operatorname{Ext}_{I}^{1}(F, T / \operatorname{soc}(T))=0 .
$$

Thus it is sufficient to show that $\operatorname{Ext}_{I}^{1}(F, \operatorname{soc}(T))=0$.

Since $X$ is injective,

$$
\begin{aligned}
\operatorname{Ext}_{I}^{1}(F, \operatorname{soc}(T)) & \cong \operatorname{Ext}_{I}^{1}(F, X \oplus Y) \\
& \cong \operatorname{Ext}_{I}^{1}(F, X) \oplus \operatorname{Ext}_{I}^{1}(F, Y) \simeq \operatorname{Ext}_{I}^{1}(F, Y)
\end{aligned}
$$


From [5, Theorem 5.2], it follows that $F$ is a flat $I$-module. Since $I / M$ is a division ring, then $\operatorname{Ext}_{I}^{1}(F, \operatorname{soc}(T)) \cong \operatorname{Ext}_{I}^{1}(F, Y) \cong \operatorname{Ext}_{I / M}^{1}\left(I / M \otimes_{I} F, Y\right)=$ 0 by [2, VI, Proposition 4.1.3]. Hence $I$ is a splitting ring.

Let

$$
N=\left\{\left(\begin{array}{lll}
a & b & c \\
0 & d & e \\
0 & 0 & f
\end{array}\right) \mid d \in M ; a, b, c, e, f \in I / M\right\}
$$

and let $U$ be the simple $R$-module $R / N$.

LEMMA 3. $\operatorname{Ext}_{\Lambda}^{n}(R, U)=0$ for $n \geqq 1$.

Proof. Since $\Lambda$ is a hereditary ring, $\operatorname{Ext}_{\Lambda}^{n}(R, U)=0$ for $n \geqq 2$. As a left $\Lambda$-module, $R=\oplus \sum\left\{\Lambda e_{i j} \mid 1 \leqq i \leqq j \leqq 3\right\}$. Since $\Lambda e_{11}, \Lambda e_{12}, \Lambda e_{13}, \Lambda e_{22}$, $\Lambda e_{33}$ are $\Lambda$-projective, then

$$
\operatorname{Ext}_{\Lambda}^{1}(R, U) \cong \oplus \sum\left\{\operatorname{Ext}_{\Lambda}^{1}\left(\Lambda e_{i j}, U\right) \mid 1 \leqq i \leqq j \leqq 3\right\} \cong \operatorname{Ext}_{\Lambda}^{1}\left(\Lambda e_{23}, U\right) .
$$

Let $0 \rightarrow U \rightarrow X \stackrel{\varphi}{\rightarrow} \Lambda e_{23} \rightarrow 0$ be an exact sequence of $\Lambda$-modules. Since $e_{11} \varphi(X)=e_{11} U=0, e_{11} X=0$. Similarly $e_{33} X=0$. Thus $X$ has identical $\Lambda$ and $I$-module structures, namely, $X \cong U \oplus \Lambda e_{23}$ (since $E\left(S_{2}\right) / S_{2} \cong S_{1}$ and $S_{2} \cong U \cong \Lambda e_{23}$ as $I$-modules). Therefore $\operatorname{Ext}_{\Lambda}^{1}\left(\Lambda e_{23}, U\right)=0$.

LEMMA 4. $\operatorname{Hom}_{\Lambda}(R, U) \cong U \oplus U$.

Proof. $\Lambda e_{11}, \Lambda e_{12}, \Lambda e_{13}$, and $\Lambda e_{33}$ are nonsingular simple $\Lambda$-modules, and $U$ is a singular simple $\Lambda$-module. Therefore

$$
\operatorname{Hom}_{\Lambda}\left(\Lambda e_{11} \oplus \Lambda e_{12} \oplus \Lambda e_{13} \oplus \Lambda e_{33}, U\right)==0 .
$$

Since $\Lambda e_{22} \cong I$ and $\Lambda e_{23} \cong U$ each have identical $\Lambda$ - and $I$-module structures and since ${ }_{I} U$ is annihilated by $M$, then

$$
\begin{aligned}
\operatorname{Hom}_{\Lambda}(R, U) & \cong \operatorname{Hom}_{\Lambda}\left(\Lambda e_{22} \oplus \Lambda e_{23}, U\right) \cong \operatorname{Hom}_{I}(I \oplus U, U) \\
& \cong \operatorname{Hom}_{I}(I, U) \oplus \operatorname{Hom}_{I}(U, U) \cong U \oplus U .
\end{aligned}
$$

Lemma 5. $\operatorname{Ext}_{R}^{n}(A, U \oplus U) \cong \operatorname{Ext}_{\Lambda}^{n}(A, U)$ for all $R$-modules $A$ and for all $n \geqq 1$.

Proof. By Lemma 3 and [2, VI, Proposition 4.1.4],

$$
\operatorname{Ext}_{R}^{n}\left(A, \operatorname{Hom}_{\Lambda}(R, U)\right) \cong \operatorname{Ext}_{\Lambda}^{n}(A, U)
$$

for all $R$-modules $A$ and for all $n \geqq 1$. Thus the conclusion follows from Lemma 4.

Lemma 6. $\operatorname{Ext}_{\Lambda}^{1}(A, U)=0$ for all nonsingular $R$-modules $A$. 
Proof. Since $\Lambda \cong I / M \oplus I / M \oplus I$, it follows from Lemma 2 that $\Lambda$ is a splitting ring. Therefore, $A=Z\left({ }_{\Lambda} A\right) \oplus F$ for any nonsingular $R$-module $A$, and $\operatorname{Ext}_{\Lambda}^{1}(F, U)=0$. So it suffices to show that $\operatorname{Ext}_{\Lambda}^{1}\left(Z\left({ }_{\Lambda} A\right), U\right)=0$.

If $0 \neq t \in Z\left({ }_{\Lambda} A\right)$, then the $\Lambda$-annihilator of $t$ is

$$
\left\{\left(\begin{array}{lll}
a & 0 & 0 \\
0 & b & 0 \\
0 & 0 & c
\end{array}\right) \mid b \in M ; a, c \in I / M\right\} \text {. }
$$

For let $K$ denote the $R$-annihilator of $t$, and let $L$ be the set of elements of $I$ which appear in the second row and second column of some element of $K$. Since $0 \neq t \in Z\left({ }_{\Lambda} A\right), L$ is a nontrivial left ideal of $I$. If $m \in M$, then

$$
\left(K:\left(\begin{array}{ccc}
0 & 0 & 0 \\
0 & m & 0 \\
0 & 0 & 0
\end{array}\right)\right) \supseteq\left\{\left(\begin{array}{ccc}
x & y & z \\
0 & u & v \\
0 & 0 & w
\end{array}\right) \mid u \in(M L: m) ; x, y, z, u, v \in I / M\right\}
$$

is essential in $R$. Since $R / K$ is a nonsingular $R$-module, this forces

$$
\left(\begin{array}{lll}
0 & 0 & 0 \\
0 & m & 0 \\
0 & 0 & 0
\end{array}\right) \in K
$$

It is now easy to verify that the $\Lambda$-annihilator has the desired form.

Thus $Z\left({ }_{\Lambda} A\right)$ is 0 or a direct sum of simple $R$-modules isomorphic to $U$. As it was noted in the proof of Lemma $3, \operatorname{Ext}_{\Lambda}^{1}(U, U)=0$. Consequently $\operatorname{Ext}_{\Lambda}^{1}\left(Z\left({ }_{\Lambda} A\right), U\right)=0$.

Lemma 7. $\operatorname{Ext}_{R}^{1}(A, B)=0$, where $A$ is any nonsingular $R$-module and $B$ is any $R$-module isomorphic to a direct sum of copies of $U$.

Proof. Let $B=\oplus \sum_{\alpha \in \mathcal{\gamma}} U_{\alpha}$, where $U_{\alpha} \cong U$. Consider the exact sequence

$$
0 \rightarrow \oplus \sum_{\alpha \in \mathcal{A}} U_{\alpha} \stackrel{f}{\rightarrow} \prod_{\alpha \in \mathcal{A}} U_{\alpha} \rightarrow \prod_{\alpha \in \mathcal{L}} U_{\alpha} / \oplus \sum_{\alpha \in \mathcal{\mathcal { H }}} U_{\alpha} \rightarrow 0 .
$$

By Lemmas 5 and $6, \operatorname{Ext}_{R}^{1}\left(A, \prod_{x \in \mathcal{L}} U_{\alpha}\right) \cong \prod_{x \in \mathcal{S}} \operatorname{Ext}_{R}^{1}\left(A, U_{\alpha}\right)=0$. Thus (*) induces an exact sequence

$$
\begin{aligned}
\operatorname{Hom}_{R}\left(A, \prod_{\alpha \in \mathscr{L}} U_{\alpha}\right) & \stackrel{f_{*}}{\longrightarrow} \operatorname{Hom}_{l i}\left(A, \prod_{\alpha \in: \mathcal{L}} U_{\alpha} / \oplus \sum_{\alpha \in \mathscr{S}} U_{\alpha}\right) \\
& \longrightarrow \operatorname{Ext}_{R R}^{1}\left(A, \oplus \sum_{\alpha \in \mathscr{A}} U_{\alpha}\right) \rightarrow 0 .
\end{aligned}
$$

Since $N U=0$, then $(*)$ is also an exact sequence of $R / N$-modules. Since 
$R / N$ is a division ring, (*) splits as $R / N$-modules and as $R$-modules. Therefore $f_{*}$ is an epimorphism, and hence $\operatorname{Ext}_{R}^{1}\left(A, \oplus \sum_{\alpha \in \mathscr{S}} U_{\alpha}\right)=\operatorname{Ext}_{R}^{1}(A, B)=$ 0 by exactness.

LEMMA 8. Every simple singular $R$-module $V \neq U$ is $R$-injective.

PROof. If $V$ is a simple singular $R$-module not isomorphic to $U$, then $V \cong R / T$, where $T$ is of one of the following types of maximal left ideals of $R$ :

$$
T=\left\{\left(\begin{array}{ccc}
a & b & c \\
0 & d & e \\
0 & 0 & f
\end{array}\right) \mid d \in L ; a, b, c, e, f \in I / M\right\},
$$

where $L$ is a maximal left ideal of $I$ distinct from $M$;

$$
T=\left\{\left(\begin{array}{lll}
a & b & c \\
0 & d & e \\
0 & 0 & 0
\end{array}\right) \mid d \in I ; a, b, c, e \in I / M\right\} .
$$

To establish that $V$ is injective, it is sufficient to show that for any essential left ideal $K$ of $R$ and for any diagram

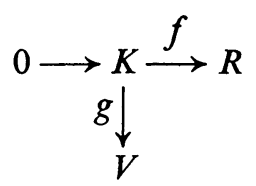

there is an $h: R \rightarrow V$ such that $h f=g$. The left ideal $K$ can be any one of the following types, where $H$ denotes a nonzero left ideal of $I$.
(A) $K=\left\{\left(\begin{array}{lll}x & y & z \\ 0 & u & 0 \\ 0 & 0 & 0\end{array}\right) \mid u \in H ; x, y, z \in I / M\right\}$.
(B) $K=\left\{\left(\begin{array}{lll}x & y & z \\ 0 & u & v \\ 0 & 0 & 0\end{array}\right) \mid u \in H ; x, y, z, v \in I / M\right\}$.
(C) $K=\left\{\left(\begin{array}{llr}x & y & z \\ 0 & u & v \\ 0 & 0 & w\end{array}\right) \mid u \in H ; x, y, z, v, w \in I / M\right\}$.

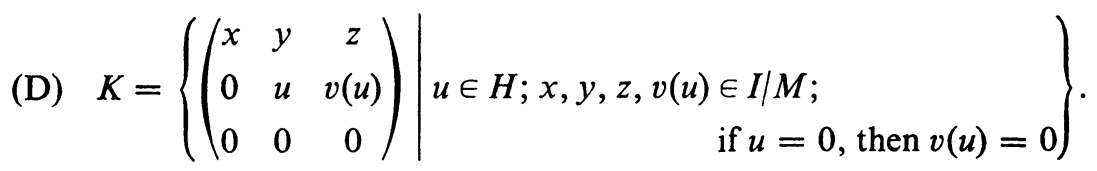


If $K$ is of type (D), the situation can be reduced to type (C) by the following argument. Let

Then

$$
P=\left\{\left(\begin{array}{lll}
0 & 0 & a \\
0 & 0 & b \\
0 & 0 & c
\end{array}\right) \mid a, b, c \in I / M\right\}
$$

$$
\left(\begin{array}{ccc}
x & y & z \\
0 & u & s \\
0 & 0 & 0
\end{array}\right)=\left(\begin{array}{ccc}
x & y & z \\
0 & u & v(u) \\
0 & 0 & 0
\end{array}\right)+\left(\begin{array}{ccc}
0 & 0 & 0 \\
0 & 0 & s-v(u) \\
0 & 0 & 0
\end{array}\right) \in K+P .
$$

Therefore $K+P$ is of type (C). Since $Z\left({ }_{R} V\right)=V$ and since $\operatorname{soc}(K)$ is nonsingular by Lemma $1(\mathrm{~b})$, then $K \cap P \subseteq \operatorname{soc}(K) \subseteq \operatorname{ker} g$. Hence $g$ can be extended to $K+P$ by setting $g(P)=0$. Therefore the situation is reduced to type (C).

Let $T$ be of type (a). If $K$ is of type (A), (B) or (C), then $g$ can be extended to all of $R$ by using the fact that $V$ is an injective $I$-module.

If $T$ is of type (b) and $K$ is of type (A) or (B), then $e_{33} K=0$ and $e_{33} V \neq 0$. Thus $g(K)=0$, and the zero map extends $g$.

Finally, if $T$ is of type (b) and $K$ is of type (C), then $g$ can be extended to $h: R \rightarrow V$ by setting $h(x)=g\left(e_{33} x\right)$ for each $x \in R$.

THEOREM 9. $R$ is a splitting ring with $1 . g 1 . \operatorname{dim} R=2$.

Proof. To show $R$ is a splitting ring, it is sufficient to show that $\operatorname{Ext}_{R}^{1}(F, T)=0$ for any nonsingular $R$-module $F$ and for any singular $R$-module $T$. By Lemma 1(a), Lemma 8 , and the fact that $N^{2}=N$, $T / \operatorname{soc}(T)$ is an injective, semisimple $R$-module. As in the proof of Lemma 2 , it can be assumed that $\operatorname{soc}(T)$ is a direct sum of copies of $U$. By Lemma $7, \operatorname{Ext}_{R}^{1}(F, \operatorname{soc}(T))=0$. From the exact sequence

$$
0=\operatorname{Ext}_{R}^{1}(F, \operatorname{soc}(T)) \rightarrow \operatorname{Ext}_{R}^{1}(F, T) \rightarrow \operatorname{Ext}_{R}^{1}(F, T / \operatorname{soc}(T))=0,
$$

it follows that $\operatorname{Ext}_{R}^{1}(F, T)=0$. Therefore $R$ is a splitting ring.

Since any splitting ring $R$ satisfies $Z\left({ }_{R} R\right)=0$ [3], then Lemma $1(\mathrm{c})$ and [7, Theorem 2.2] yield l.gl.dim $R=2$.

The authors are grateful to J. Kuzmanovich for several stimulating letters and conversations related to splitting rings.

\section{REFERENCES}

1. J. S. Alin and S. E. Dickson, Goldie's torsion theory and its derived functor, Pacific J. Math. 24 (1968), 195-293. MR 37 \#2834.

2. H. Cartan and S. Eilenberg, Homological algebra, Princeton Univ. Press, Princeton, N.J., 1956. MR 17, 1040. 
3. V. C. Cateforis and F. L. Sandomierski, The singular submodule splits off, J. Algebra 10 (1968), 149-165. MR 39 \#2805.

4. J. H. Cozzens, Homological properties of the ring of differential polynomials, Bull. Amer. Math. Soc. 76 (1970), 75-79. MR 41 \#3531.

5. L. Levy, Torsion-free and divisible modules over non-integral domains, Canad. J. Math. 15 (1963), 132-151. MR 26 \#155.

6. J. C. Robson, Idealizers and hereditary Noetherian prime rings, J. Algebra (to appear).

7. M. L. Teply, Homological dimension and splitting torsion theories, Pacific J. Math. 34 (1970), 193-205. MR 43 \#291.

8. D. B. Webber, Ideals and modules of simple Noetherian hereditary rings, J. Algebra 16 (1970), 239-242. MR 42 \#305.

Department of Mathematics, University of Florida, Gainesville, Florida 32601

Current address (John D. Fuelberth): Department of Mathematics, University of Northern Colorado, Greeley, Colorado 80631 